\title{
Neoadjuvant FOLFIRINOX in Patients With Borderline Resectable Pancreatic Cancer: A Systematic Review and Patient-Level Meta-Analysis
}

\author{
Quisette P. Janssen, Stefan Buettner, Mustafa Suker, Berend R. Beumer, Pietro Addeo, \\ Philippe Bachellier, Nathan Bahary, Tanios Bekaii-Saab, Maria A. Bali, Marc G. Besselink, \\ Brian A. Boone, Ian Chau, Stephen Clarke, Mary Dillhoff, Bassel F. El-Rayes, Jessica M. \\ Frakes, Derek Grose, Peter J. Hosein, Nigel B. Jamieson, Ammar A. Javed, Khurum Khan, \\ Kyu-Pyo Kim, Song Cheol Kim, Sunhee S. Kim, Andrew H. Ko, Jill Lacy, Georgios A. \\ Margonis, Martin D. McCarter, Colin J. McKay, Eric A. Mellon, Sing Yu Moorcraft, Ken-Ichi \\ Okada, Alessandro Paniccia, Parag J. Parikh, Niek A. Peters, Hans Rabl, Jaswinder Samra, \\ Christoph Tinchon, Geertjan van Tienhoven, Eran van Veldhuisen, Andrea Wang-Gillam, \\ Matthew J. Weiss, Johanna W. Wilmink, Hiroki Yamaue, Marjolein Y. V. Homs, \\ Casper H. J. van Eijck, Matthew H. G. Katz, Bas Groot Koerkamp
}

\begin{abstract}
Background: FOLFIRINOX is a standard treatment for metastatic pancreatic cancer patients. The effectiveness of neoadjuvant FOLFIRINOX in patients with borderline resectable pancreatic cancer (BRPC) remains debated.

Methods: We performed a systematic review and patient-level meta-analysis on neoadjuvant FOLFIRINOX in patients with BRPC. Studies with BRPC patients who received FOLFIRINOX as first-line neoadjuvant treatment were included. The primary endpoint was overall survival (OS), and secondary endpoints were progression-free survival, resection rate, R0 resection rate, and grade III-IV adverse events. Patient-level survival outcomes were obtained from authors of the included studies and analyzed using the Kaplan-Meier method.

Results: We included 24 studies (8 prospective, 16 retrospective), comprising 313 (38.1\%) BRPC patients treated with FOLFIRINOX. Most studies $(\mathrm{n}=20)$ presented intention-to-treat results. The median number of administered neoadjuvant FOLFIRINOX cycles ranged from 4 to 9 . The resection rate was $67.8 \%(95 \%$ confidence interval $[\mathrm{CI}]=60.1 \%$ to $74.6 \%)$, and the R0-resection rate was $83.9 \%$ (95\% CI $=76.8 \%$ to $89.1 \%)$. The median OS varied from 11.0 to 34.2 months across studies. Patientlevel survival data were obtained for 20 studies representing 283 BRPC patients. The patient-level median OS was 22.2 months ( $95 \% \mathrm{CI}=18.8$ to 25.6 months), and patient-level median progression-free survival was 18.0 months $(95 \% \mathrm{CI}=14.5$ to 21.5 months). Pooled event rates for grade III-IV adverse events were highest for neutropenia (17.5 per 100 patients, $95 \% \mathrm{CI}=$ $10.3 \%$ to $28.3 \%$ ), diarrhea (11.1 per 100 patients, $95 \% \mathrm{CI}=8.6$ to 14.3 ), and fatigue (10.8 per 100 patients, $95 \% \mathrm{CI}=8.1$ to 14.2 ). No deaths were attributed to FOLFIRINOX.
\end{abstract}


Conclusions: This patient-level meta-analysis of BRPC patients treated with neoadjuvant FOLFIRINOX showed a favorable median OS, resection rate, and R0-resection rate. These results need to be assessed in a randomized trial.

Pancreatic cancer is expected to be the second-leading cause of cancer-related death by 2030 (1). Approximately $20 \%$ of patients have borderline resectable pancreatic cancer (BRPC) or upfront resectable pancreatic cancer at diagnosis (2). Even after curative-intent surgery, cure is exceedingly rare, as demonstrated by a 10 -year overall survival (OS) of $4 \%$ (3). Upfront resection with adjuvant chemotherapy has long been the standard of care for patients with localized pancreatic cancer. However, due to postoperative complications, deteriorating performance status, and early progressive disease, only about $55 \%$ of patients receive adjuvant chemotherapy (4-6). With a neoadjuvant approach, almost all patients receive systemic chemotherapy. This approach aims to address occult metastatic disease, increase the rate of Ro resection, and avoid futile surgery in patients with rapidly progressive disease (7) .

Several neoadjuvant treatment regimens with or without chemoradiotherapy (CRT) have been proposed for BRPC patients (8-10). A combination chemotherapy regimen of folinic acid (leucovorin), fluorouracil (5-FU), irinotecan, and oxaliplatin (FOLFIRINOX) seems to be the most effective regimen for patients with pancreatic cancer. In a randomized, controlled trial (RCT), patients with metastatic pancreatic cancer had a superior OS with FOLFIRINOX compared with gemcitabine (median 11.1 vs 6.8 months, $P<.001)$ (11). No RCT has been published with FOLFIRINOX in the neoadjuvant setting for BRPC patients. All published phase I-II trials and cohort studies on neoadjuvant FOLFIRINOX for BRPC patients are small and therefore report a wide range of median OS (12-15).

The primary aim of this systematic review and patient-level meta-analysis was to determine OS after neoadjuvant FOLFIRINOX as first-line treatment for patients with BRPC. Secondary outcomes included progression-free survival (PFS), resection rate, $\mathrm{RO}$ resection rate, and grade III-IV adverse events (AEs).

\section{Methods}

\section{Eligibility}

We searched for studies containing treatment-naïve patients with BRPC treated with FOLFIRINOX as neoadjuvant therapy, irrespective of further treatment after FOLFIRINOX. Case reports, reviews, letters to the editor, conference abstracts without full text, and studies only reporting on specific groups of patients (eg, only patients in a specific age group) were excluded.

\section{Search Strategy}

This systematic review was performed in accordance with the Preferred Reporting Items for Systematic Reviews and MetaAnalysis standard guidelines (16). To identify relevant studies, a comprehensive librarian-led search of Embase, MEDLINE (via OvidSP), Web of Science, Scopus, Cochrane Central, and Google Scholar was performed on September 1, 2017. Search terms included "FOLFIRINOX," "folinic acid," "fluorouracil," "irinotecan," "oxaliplatin," "pancreas cancer," "drug combination," and relevant variants thereof. Only articles written in English were assessed. No restrictions on publication date were applied.
Literature without formal publication was not assessed. A full description of the search is summarized in the Supplementary Methods (available online).

\section{Selection Procedure and Data Collection}

After removal of duplicates, Q. P. Janssen and S. Buettner independently reviewed the abstracts for eligibility. The full-text article of any study that met the inclusion criteria was retrieved for further assessment. Full-text studies were excluded if only a regimen other than FOLFIRINOX was used, if the study did not include at least one BRPC patient, if the study was not an original report, or if the same patient cohort was presented in another study. Disagreements were resolved through discussion and consensus. Q. P. Janssen and S. Buettner extracted the data from selected studies with use of standardized data collection forms. The primary outcome was OS. Secondary outcomes were PFS, resection rates, R0 resection rates, and Common Terminology Criteria for Adverse Events grade III-IV (17). Other collected data included study characteristics (first author, year of publication, study design, inclusion period, duration of follow-up), study population specifications (total sample size, number of patients treated with FOLFIRINOX in total and per disease stage), and details on the intervention (FOLFIRINOX regimen, number of administered cycles, other treatments).

For the patient-level meta-analysis, we contacted the authors of all studies to obtain (updated) patient-level data on OS and PFS. Data were collected for BRPC patients only. The authors of four studies $(14,18-20)$ provided patient-level data for additional BRPC patients not included in the reviewed articles. Data other than OS and PFS were not collected at the patient level but reported as aggregate outcomes from the published studies.

\section{Methodological Assessment}

All studies were assessed for risk of bias using an appraisal system developed by the Critical Appraisal Skill Program (21). This critical appraisal tool is designed to systematically assess the methodology of individual studies. Publication bias was assessed with a funnel plot (22).

\section{Statistical Analysis}

Patient-level survival outcomes were analyzed with the KaplanMeier method using the $r m s$ and survival packages for $\mathrm{R}$ 3.5.0 (https://cran.r-project.org/). The Kaplan-Meier method was used to account for censoring of patients alive or without recurrence at last follow-up. The primary survival outcome was OS, and the secondary outcome was PFS. Median, 1-year, 3-year, and 5year survival were analyzed and reported for OS, and median, 1year, 2-year, and 3-year for PFS. Patient-level survival outcomes were calculated from treatment initiation. One study only reported the date of surgery; therefore, 11 weeks were added to the date of surgery to account for a median of four cycles of FOLFIRINOX (8 weeks) with an additional 3-week interval to surgery (18). We performed post-hoc sensitivity analyses on patient-level survival data after exclusion of studies including 
only patients who underwent a resection after neoadjuvant therapy, comparing retrospective and prospective studies, comparing studies in which the number of FOLFIRINOX cycles was at least six or less than six, comparing studies using full-dose or modified FOLFIRINOX regimens, comparing studies with or without granulocyte-colony stimulating factor (G-CSF) primary prophylaxis, analyzing the influence of (neo)adjuvant (chemo)radiation therapy after neoadjuvant FOLFIRINOX on survival, and including only patients who were recurrence-free after 12 months. Survival distributions were compared using the logrank test. The 95\% confidence intervals (CI) for survival estimates at 1, 2, 3, and 5 years were calculated using a $\log (-\log$ $\hat{\mathrm{S}}(\mathrm{t}))$ transformation.

Pooled proportions of resection and R0 resection were calculated. The $\mathrm{I}^{2}$ statistic was estimated for both proportions to assess whether observed differences in proportions were compatible with chance alone or partly attributable to heterogeneity. The $\mathrm{I}^{2}$ statistic estimates the percentage of variation across studies that can be ascribed to heterogeneity rather than chance (23). An $\mathrm{I}^{2}$ above $50 \%$ is considered substantial heterogeneity (24). Random-effects models rather than fixed-effects models were used because heterogeneity in the definitions of disease stage across studies was anticipated to cause heterogeneity in the proportion of resection and R0 resection (23). Studies only reporting data for BRPC patients who underwent a resection after neoadjuvant FOLFIRINOX were included only for the analysis of $\mathrm{R} 0$ resection rates, not for overall resection rates. Grade III or IV AEs were calculated as number of events per 100 patients and pooled in random-effects models. AEs were pooled separately for prospective and retrospective studies. We performed a subgroup analysis comparing grade III or IV event rates of neutropenia and febrile neutropenia in studies with or without G-CSF prophylaxis. Pooled analyses were performed using the meta package for $R$ 3.5.0. All tests were two-sided. A P value less than .05 was considered statistically significant.

\section{Results}

\section{Included Studies}

We identified 2659 potentially relevant studies. Based on the abstracts, 54 studies were selected for full-text assessment, of which 24 studies (representing 1802 patients) fulfilled all inclusion criteria (Figure 1) . Ten studies were excluded because only regimens other than FOLFIRINOX were used, 15 studies because no BRPC patients were included, 2 studies because the article was written in a language other than English, 1 study was a protocol, and 2 studies overlapped with other included studies (Supplementary Methods, available online).

Table 1 shows the study characteristics. Resectability status was defined by National Comprehensive Cancer Network criteria (42) in eight studies, Americas Hepato-Pancreato-Biliary Association/Society of Surgical Oncology/Society for Surgery of the Alimentary Tract criteria (43) in seven studies, the ALLIANCE criteria (44) in two studies, and other criteria $(26,45-$ 47) in four studies. Three studies did not report staging criteria. Most studies $(\mathrm{n}=20)$ presented intention-to-treat results of all BRPC patients who started with neoadjuvant FOLFIRINOX, regardless of subsequent resection. Four studies included only patients who underwent a resection after neoadjuvant treatment $(12,15,20,32)$. Eight studies included only patients with BRPC, 11 studies combined BRPC and locally advanced pancreatic cancer (LAPC) patients, and five studies combined all disease stages. For 89 patients in three studies, no distinction could be made between BRPC or LAPC; therefore, their results were only used for AEs and in patient-level analyses if BRPC was confirmed by the authors $(32,35,36)$.

FOLFIRINOX was given to 822 (45.6\%) patients, of whom 313 (38.1\%) patients were staged as BRPC. Only nine patients (1.1\%) from two studies had resectable pancreatic cancer (Table 1) $(12,20)$. Patient-level data were obtained from 20 studies reflecting 283 BRPC patients, representing $90.4 \%$ of all published BRPC patients who received neoadjuvant FOLFIRINOX.

\section{Methodological Assessment}

We included eight prospective and 16 retrospective studies. Six studies were multicenter studies (Table 1). Results of the methodological assessment of all included studies are reported in Supplementary Table 1 (available online). The funnel plot showed no evidence of publication bias among the included studies (Supplementary Figure 1, available online).

\section{Survival Analysis}

Seven studies $(12,13,20,25,28-30)$ representing 151 patients separately reported survival data for BRPC patients treated with neoadjuvant FOLFIRINOX. The median OS for BRPC patients varied across these seven studies from 11.0 to 34.2 months, and the median PFS varied from 5.7 to 21.3 months (Supplementary Table 2, available online).

Patient-level data were obtained for 283 BRPC patients who received neoadjuvant FOLFIRINOX, of whom 168 (59.4\%) died during follow-up. The median follow-up of patients alive at last follow-up was 22.9 months. The overall patient-level median OS was 22.2 months ( $95 \% \mathrm{CI}=18.8$ to 25.6 months) (Figure $2 \mathrm{~A})$. The pooled OS at 1 year was $76.0 \%$ ( $95 \%$ CI $=70.3 \%$ to $80.7 \%$ ), at 3 years $36.2 \%(95 \% \mathrm{CI}=29.8 \%$ to $42.6 \%$ ), and at 5 years $21.2 \%$ (95\% CI $=14.6 \%$ to $28.6 \%$ ). A total 115 of 283 patients $(40.6 \%)$ were censored. After excluding 21 patients from two studies $(20,32)$ that included only patients who underwent a resection, the patient-level median OS was similar $(22.2$ months, $95 \% \mathrm{CI}=$ 18.8 to 25.7 months, $P=.79$ ). No statistically significant difference was observed when comparing OS of patients in prospective (21.7 months, 95\% CI $=17.9$ to 25.6 months) and retrospective studies (22.4 months, 95\% CI $=17.7$ to 27.2 months) $(P=.36)$. For patients who were recurrence-free after 12 months, median OS was 43.2 months $(95 \% \mathrm{CI}=37.0$ to 49.4 months).

For studies in which patients received a median number of FOLFIRINOX cycles of six or fewer, the median OS was 21.4 months $(95 \% \mathrm{CI}=16.7$ to 26.0 months) compared with 21.7 months ( $95 \% \mathrm{CI}=15.0$ to 28.4 months) for patients in studies with a median of less than six cycles $(P=.46)$ (data not shown). No statistically significant association was found between the reported median number of FOLFIRINOX cycles administered and the patient-level median OS (Supplementary Figure 2, available online; $P=.05$ ). The median OS without upfront dose modification of FOLFIRINOX was 25.0 months $(95 \%$ $\mathrm{CI}=18.7$ to 31.2 months) compared with 21.7 months (95\% CI = 17.1 to 26.4 months) in studies with any dose modification $(P=.18)$. The median OS in studies with G-CSF prophylaxis was 20.8 months (95\% CI $=17.2$ to 24.4 months) compared with a median OS of 18.5 months ( $95 \% \mathrm{CI}=13.2$ to 23.8 months) in studies in which G-CSF was prescribed at the discretion of the treating physician $(P=.42)$. 


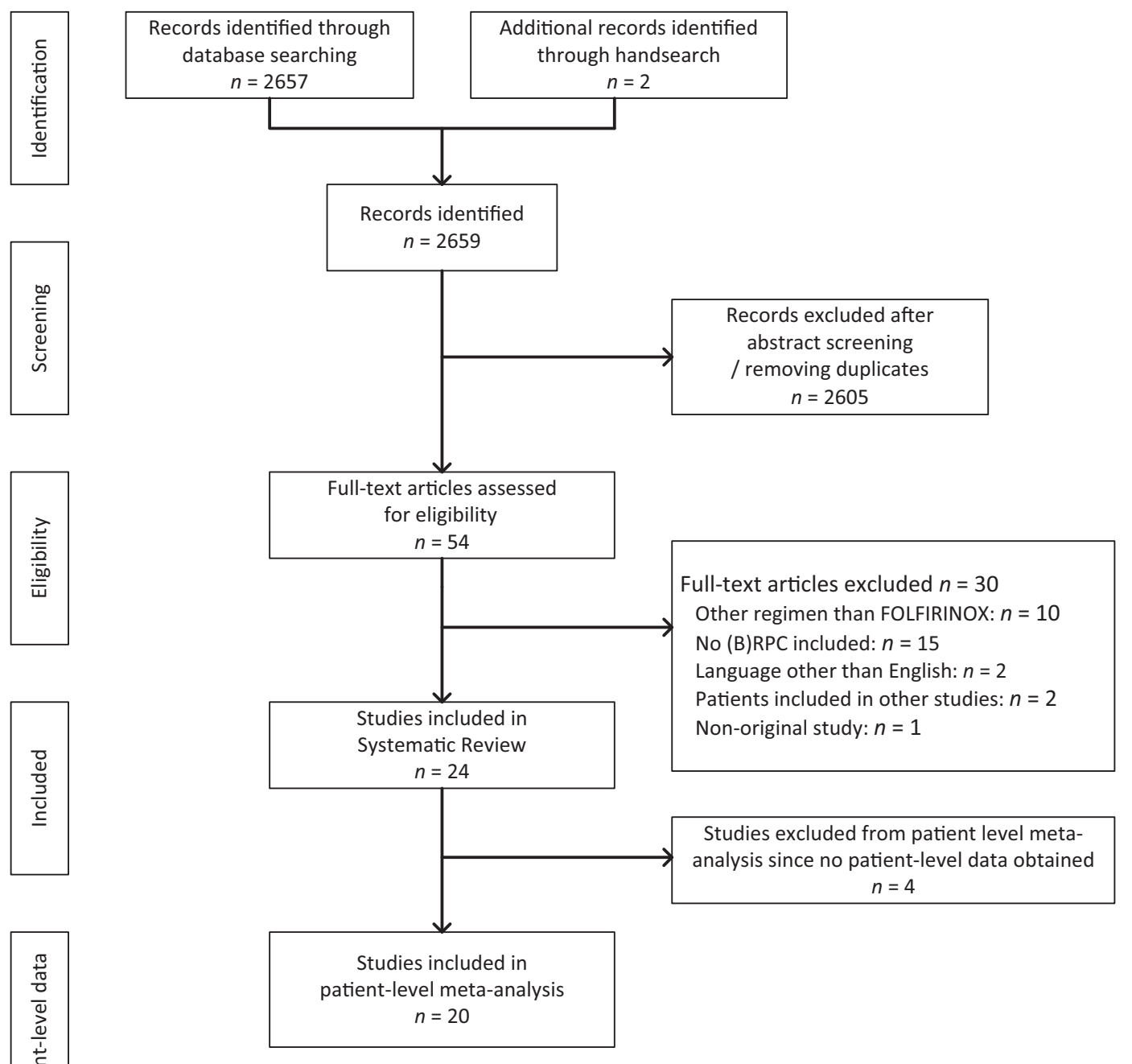

Figure 1. PRISMA flow chart showing selection of articles for systematic review and meta-analysis. (B)RPC $=($ borderline) resectable pancreatic cancer.

Patient-level PFS was available for 237 BRPC patients (20 studies), of whom 144 patients $(59.8 \%)$ showed progression or died during follow-up, with a median PFS of 18.0 months $(95 \%$ $\mathrm{CI}=14.5$ to 21.5 months; Figure $2 \mathrm{~B}$ ). A total 93 of 237 patients $(39.2 \%)$ were censored. After excluding the two studies $(20,32)$ reporting only patients who underwent a resection $(n=13)$, the median PFS was 18.0 months $(95 \% \mathrm{CI}=14.6$ to 21.4 months, $P=.99)$. The PFS at 1 year was $68.5 \%(95 \% \mathrm{CI}=61.85 \%$ to $74.4 \%)$, at 2 years $39.4 \%$ (95\% CI $=32.4 \%$ to $46.4 \%$ ), and at 3 years $25.8 \%$ ( $95 \% \mathrm{CI}=19.4 \%$ to $32.6 \%$ ). For prospective studies and retrospective studies, the median PFS was 18.4 months $(95 \% \mathrm{CI}=12.1$ to 24.8 months) and 17.7 months ( $95 \% \mathrm{CI}=14.4$ to 21.0 months), respectively $(P=.60)$.

\section{Chemotherapy Regimens}

Details of the chemotherapy regimen used are shown in Table 2. Only six studies reported the number of planned neoadjuvant FOLFIRINOX cycles for BRPC patients only, ranging from 4 to 8 cycles. Eleven studies reported the median number of FOLFIRINOX cycles administered to BRPC patients only, ranging from 4 to 9 cycles. Of these studies, five reported a median number of FOLFIRINOX cycles administered of six or more. Eight studies used a dose modification as compared with the FOLFIRINOX regimen described in the PRODIGE 4/ACCORD 11 trial (11); five studies did not include a fluorouracil bolus injection $(13,27,28,34,39)$, four studies used a lower dose of irinotecan $(27,34,35,41)$, three studies did not mention inclusion of leucovorin $(27,28,34)$, and one study gave fluorouracil continuous infusion with doses halved (14). Seven studies did not specify the FOLFIRINOX regimen administered yet mentioned using modified FOLFIRINOX (30), FOLFOX or FOLFIRINOX (18), or FOLFIRINOX without mentioning upfront dose modifications $(15,20,31,32,37)$. Use of G-CSF was reported as primary prophylaxis in seven studies $(13,19,28,34,37,39,41)$ and was prescribed at the discretion of the treating physician in five studies $(26,27,33,38,40)$.

\section{Adverse Events}

AEs during treatment with FOLFIRINOX were reported in 14 studies, of which nine studies reported only pooled outcomes across disease stages. In these 14 studies comprising 526 patients treated with FOLFIRINOX, 401 grade III or IV AEs were 


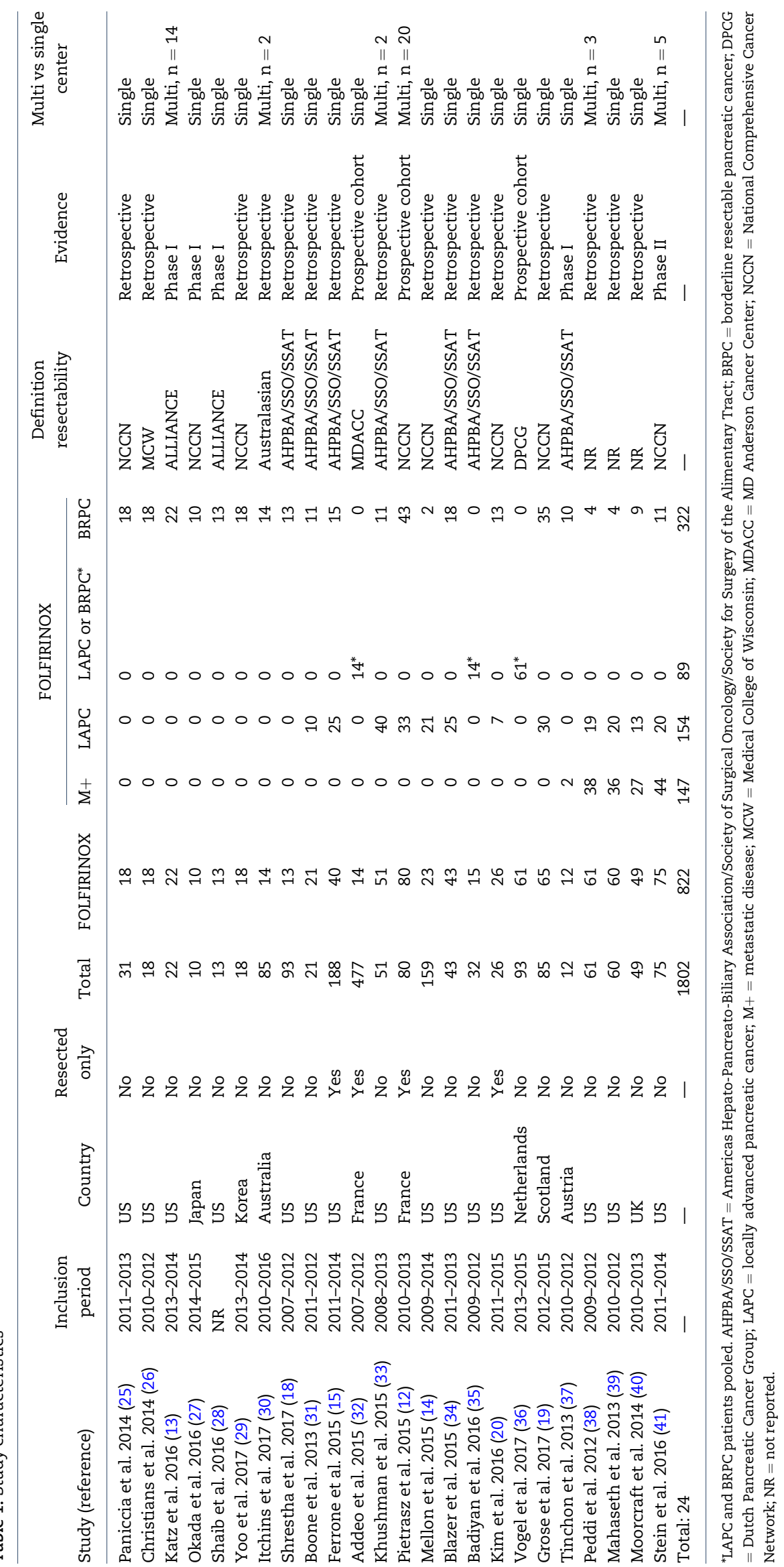




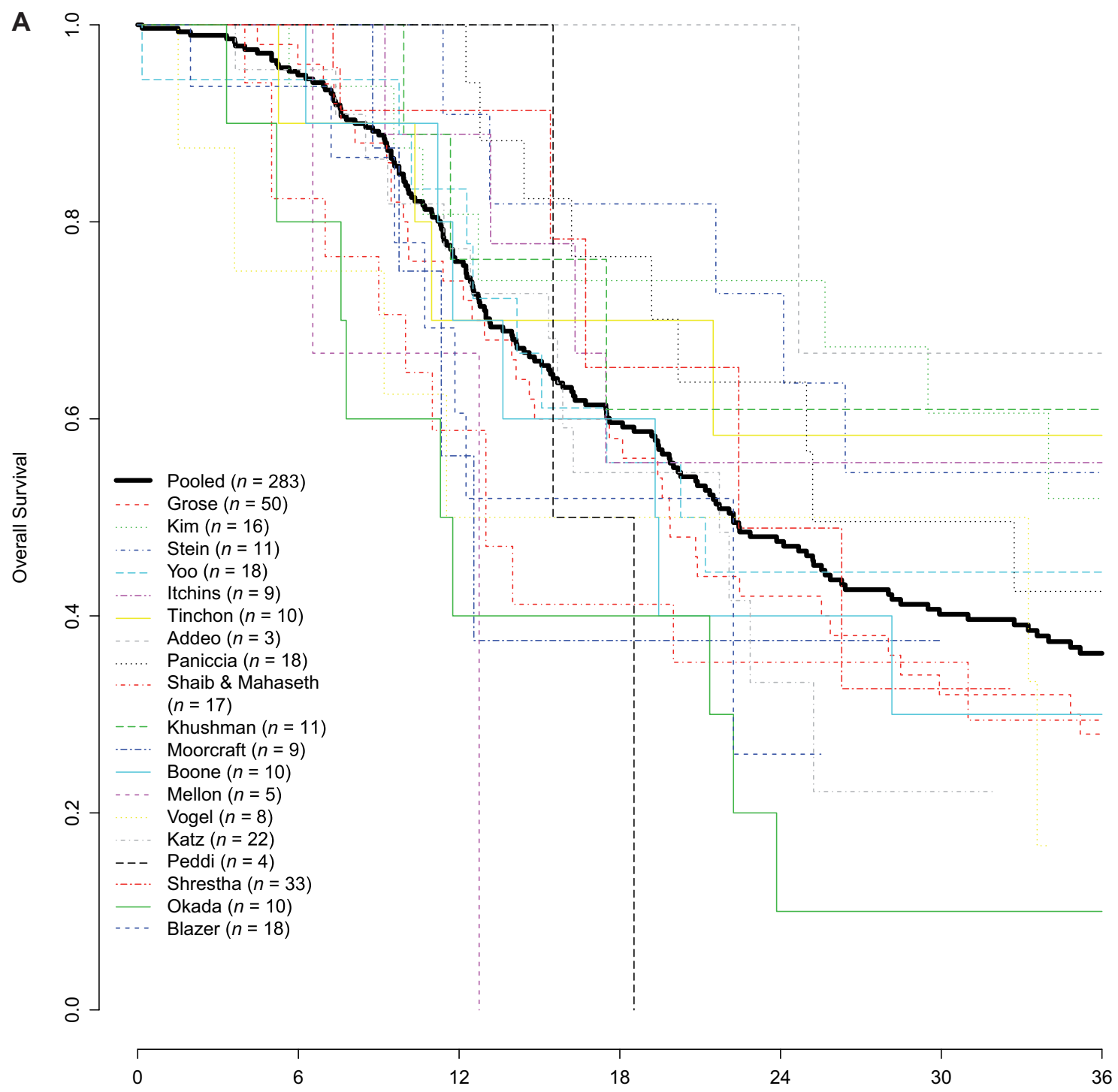

Time, months

Total Number of Events: 168

Numbers at Risk 283

253

184

132

98

78

58 Pooled

Figure 2. Pooled and patient-level overall survival (OS) and progression-free survival (PFS). Pooled and patient-level (A) OS and (B) pooled and patient-level PFS were analyzed. Patient-level survival outcomes were calculated from treatment initiation and analyzed using the Kaplan-Meier method. The Kaplan-Meier method was used to account for censoring of patients alive or without recurrence at last follow-up. Pooled median OS was $22.2 \mathrm{months}(95 \%$ confidence interval [CI] $=18.8$ to 25.6 months). Pooled median PFS was 18.0 months ( $95 \% \mathrm{CI}=14.5$ to 21.5 months).

reported (Table 3). No deaths were attributed to FOLFIRINOX. Neutropenia, diarrhea, and fatigue were the most commonly reported grade III-IV AEs. Neutropenia had a pooled event rate of 17.5 per 100 patients ( $95 \% \mathrm{CI}=10.3$ to $\left.28.3, \mathrm{I}^{2}=76.2 \%\right)$. The pooled event rates per 100 patients for other common AEs were $16.5\left(95 \% \mathrm{CI}=8.4\right.$ to $\left.29.9, \mathrm{I}^{2}=0.0 \%\right)$ for leukopenia, $10.8(95 \% \mathrm{CI}$ $=8.1$ to $\left.14.2, \mathrm{I}^{2}=0.0 \%\right)$ for fatigue, $11.1\left(95 \% \mathrm{CI}=8.6\right.$ to $14.3, \mathrm{I}^{2}=$ $0.0 \%$ ) for diarrhea, 10.4 (95\% CI $=5.5$ to $\left.18.9, \mathrm{I}^{2}=71.4 \%\right)$ for nausea or vomiting, 8.5 (95\% CI = 5.2 to $\left.13.7, \mathrm{I}^{2}=0.0 \%\right)$ for thromboembolism, and 8.9 (95\% CI $=6.2$ to $\left.12.5, \mathrm{I}^{2}=4.3 \%\right)$ for thrombocytopenia. The pooled event rate for neutropenia was lower in six studies that administered G-CSF as primary prophylaxis compared with five studies with prescription of G-CSF at the discretion of the physician (eight per 100 patients vs 23 per 100 patients, $P=.01$; forest plot in Supplementary Figure 3, available online). The results were similar for febrile neutropenia ( 3 per 100 patients vs 10 per 100 patients, $P=.02$; forest plot in Supplementary Figure 4, available online).

\section{Additional Treatment Modalities}

Several studies reported the use of CRT $(n=8)$, stereotactic body radiation therapy $(n=4)$, or intra-operative radiation therapy $(n=4)$ besides FOLFIRINOX for at least one BRPC patient (Table 2). Neoadjuvant CRT was given as standard additional 


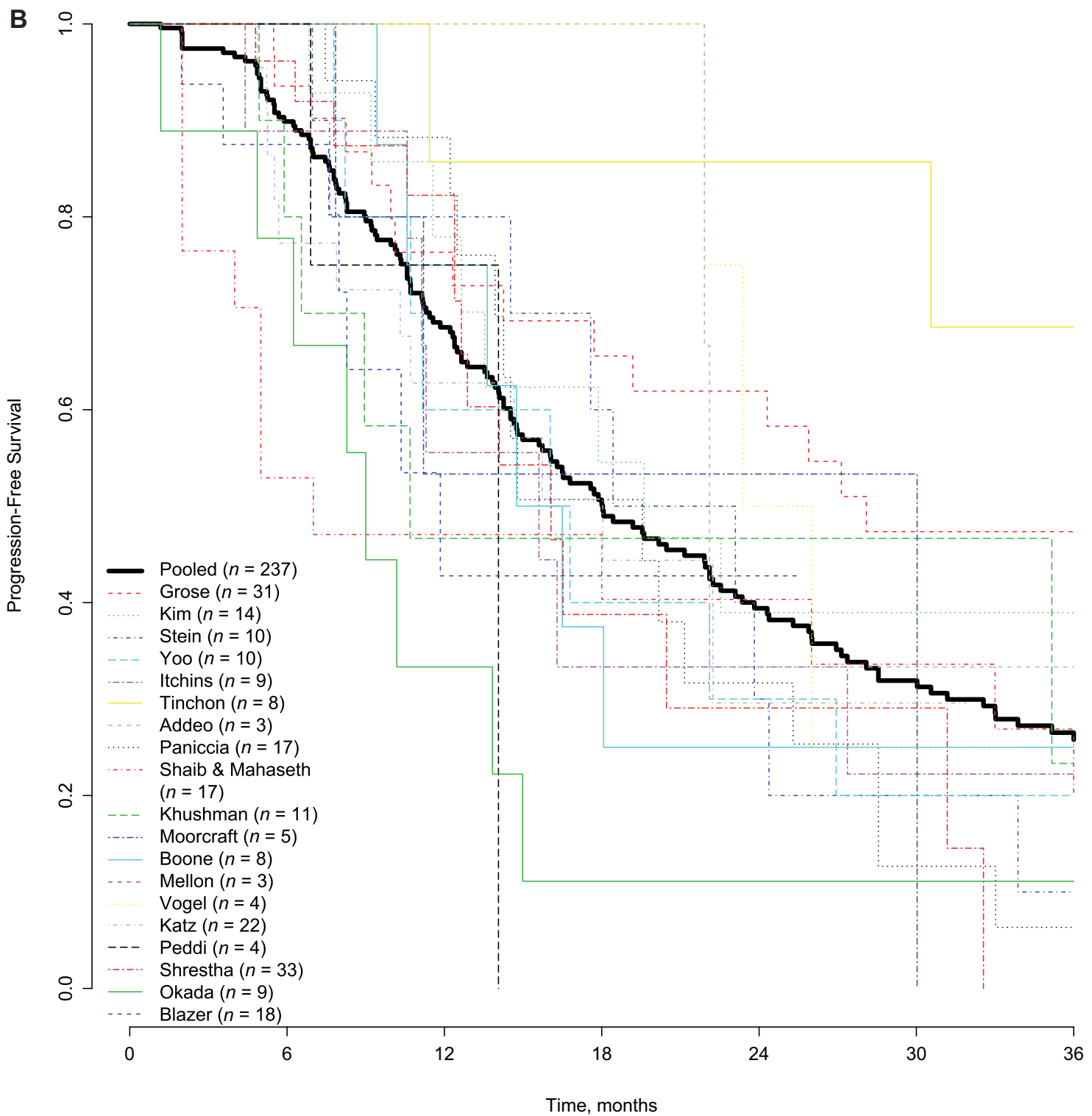

Total Number of Events: 144

Numbers at Risk 237

197

134

89

65

49

36 Pooled

Figure 2. Continued.

treatment for BRPC patients in three studies $(13,26,39)$ and reported as possible additional treatment in five other studies $(12,20,25,29,34)$. No correlation was found between the percentage of (neo)adjuvant (chemo)radiation therapy and patient-level median OS (Supplementary Figure 5, available online, $P=.14)$. Two studies were not included in this analysis because they included only patients who underwent a resection $(12,20)$.

\section{Resection and R0 Resection Rates}

Fourteen studies reported resection rates for BRPC patients treated with neoadjuvant FOLFIRINOX (Table 2). The pooled resection rate was $67.8 \%\left(95 \% \mathrm{CI}=60.1 \%\right.$ to $74.6 \%, \mathrm{I}^{2}=0.0 \%$ ). Resection margins were reported in 13 studies (Table 2). The pooled R0 resection rate in a random-effects model was $83.9 \%$ (95\% CI $=76.8 \%$ to $89.1 \%, \mathrm{I}^{2}=0.0 \%$ ).

\section{Discussion}

This patient-level meta-analysis of 20 studies representing 283 patients who received neoadjuvant FOLFIRINOX for BRPC showed a median OS of 22.2 months $(95 \% \mathrm{CI}=18.8$ to 25.6 months). After neoadjuvant FOLFIRINOX, 67.8\% (95\% CI $=60.1 \%$ to $74.6 \%$ ) of patients underwent a curative-intent resection with an $\mathrm{R} 0$ resection rate of $83.9 \%(95 \% \mathrm{CI}=76.8 \%$ to $89.1 \%)$. The rate of grade III or IV AEs was high, but no death was attributed to FOLFIRINOX.

FOLFIRINOX has been studied for patients with advanced pancreatic cancer since 2005 (48). For metastatic pancreatic 


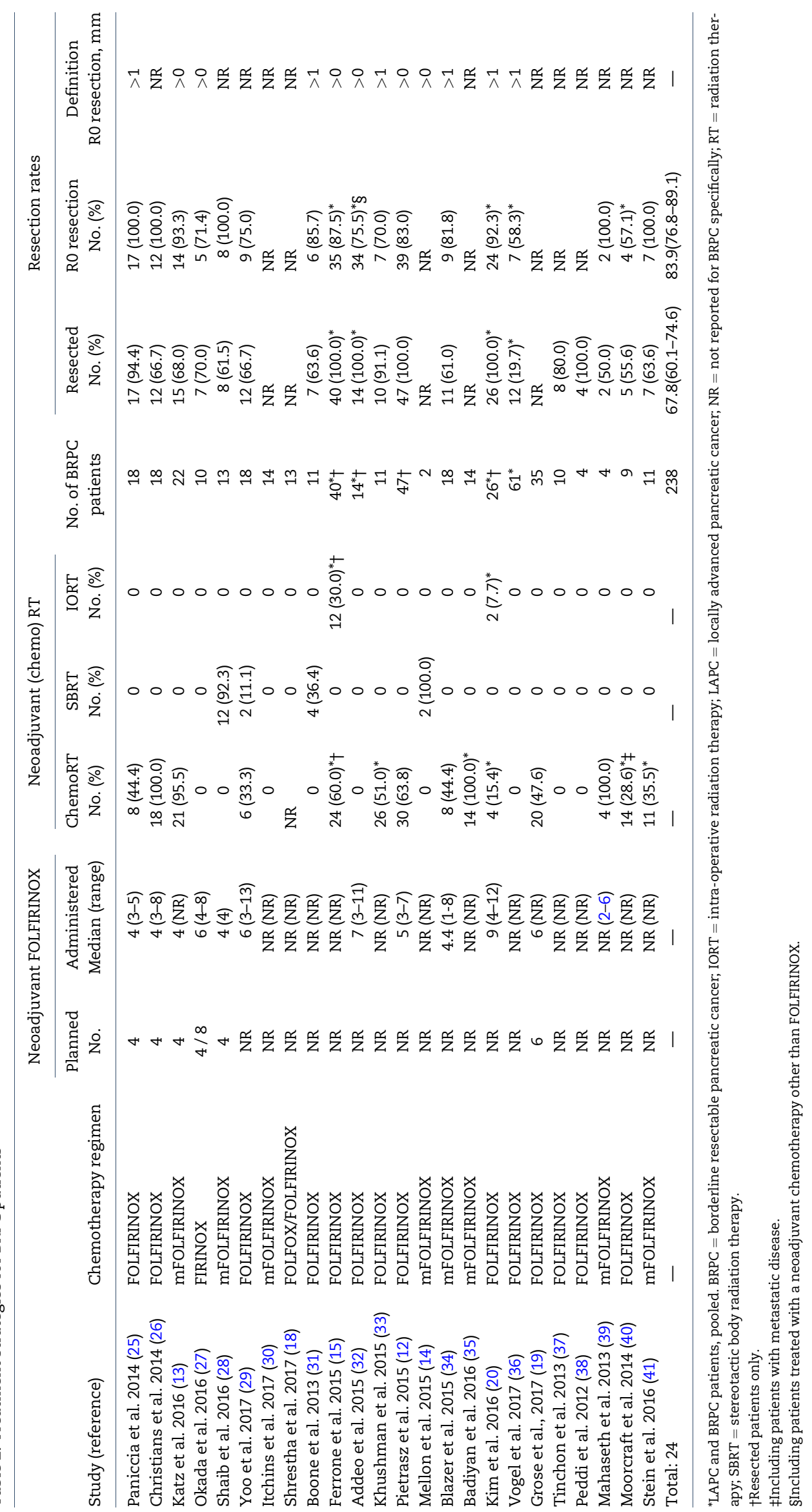




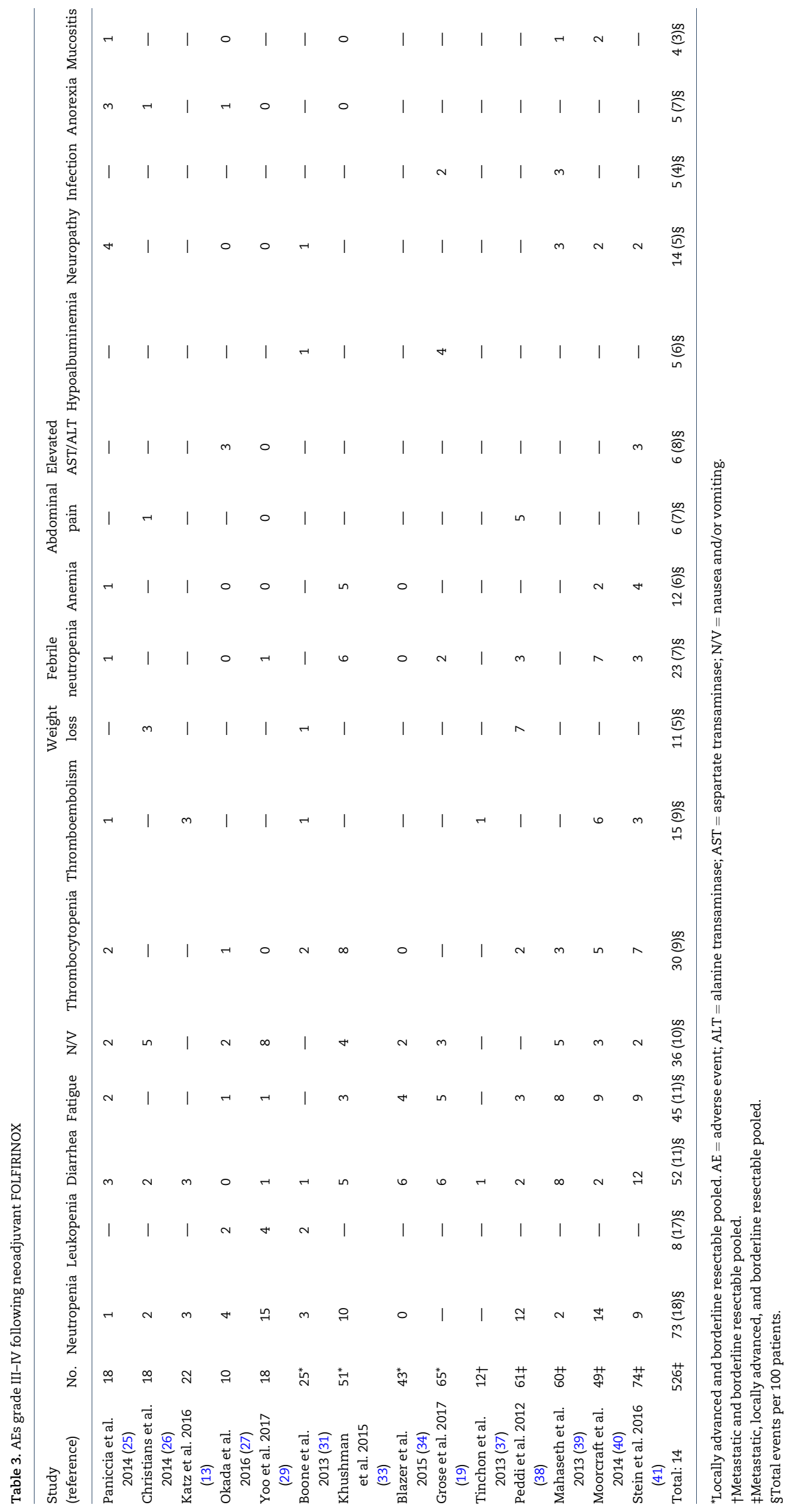




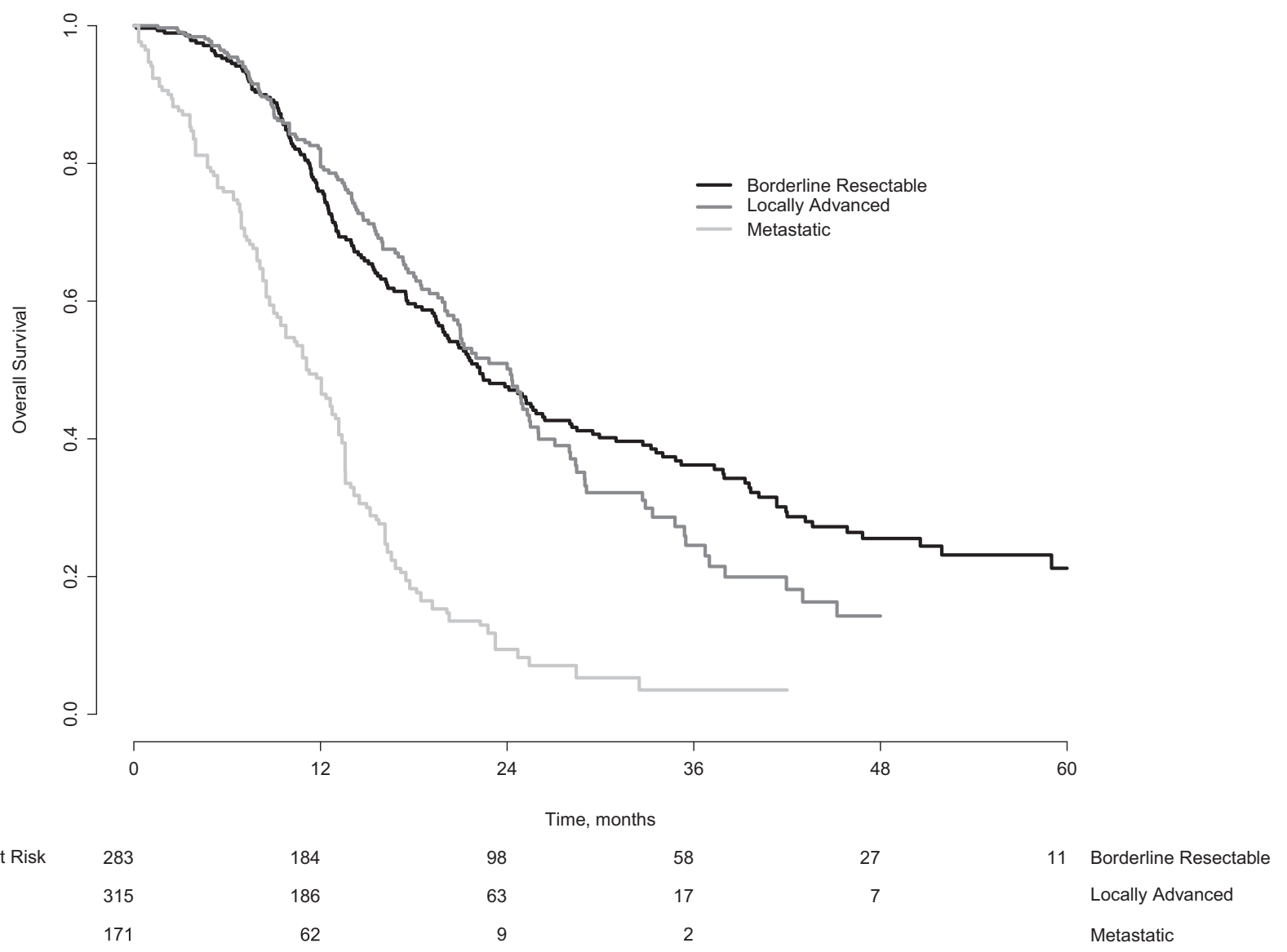

Figure 3. Pooled overall survival for patients with borderline resectable, locally advanced, and metastatic pancreatic cancer. For the survival curve of the locally advanced pancreatic cancer patients, patient-level data from the meta-analysis Suker et al. (49) was used. For the estimation of the survival curve for the metastatic pancreatic cancer patients, data were extracted from the graph of Conroy et al. (11).

cancer, palliative FOLFIRINOX has been the standard of care for patients with a good performance status since an RCT found a median OS of 11 months vs 7 months with gemcitabine (11). In patients with LAPC, no RCT has been published for induction chemotherapy with FOLFIRINOX. The best available evidence of FOLFIRINOX for LAPC is a systematic review and patient-level meta-analysis of 315 patients (11 studies) that found a median OS of 24.2 months ( $95 \% \mathrm{CI}=21.7$ to 26.8 months) (49). Figure 3 compares the patient-level OS of patients who received FOLFIRINOX in the setting of BRPC (present study) and LAPC (49). OS for both groups is clearly superior to OS for patients treated with FOLFIRINOX for metastatic pancreas cancer in the RCT of Conroy et al. (11). It is remarkable that the median OS of 22.2 months for BRPC patients in this study is similar to the 24.2 months in the LAPC setting. The survival curves of LAPC and BRPC overlap for the initial 2 years, after which they diverge. OS after 3 years was $36.2 \%$ for BRPC vs $23.0 \%$ for LAPC patients. The difference in local extent of the disease between BRPC and LAPC appears to be irrelevant for about one-half of the patients who die within 2 years. The difference in OS after 2 years probably reflects both less advanced disease and a higher resection rate for BRPC (68\% vs $27 \%$ in the LAPC setting) (49).

The use of radiation therapy after neoadjuvant FOLFIRINOX varied across studies. At the study level, no association was found between the percentage of patients who received neoadjuvant (chemo)radiation and median OS (Supplementary Figure
3, available online). Versteijne et al. performed a meta-analysis of intention-to-treat outcomes of any neoadjuvant approach vs upfront resection for (borderline) resectable pancreatic cancer [(B)RPC]. In a subgroup analysis comparing neoadjuvant approaches with and without radiation therapy, they also found no difference in OS (50). The interim analysis of the Dutch PREOPANC-1 trial, as presented at the ASCO annual meeting in 2018 , showed a twofold increase in $\mathrm{R} 0$ resection rate, with $31 \%$ after upfront resection vs $65 \%$ after neoadjuvant CRT $(P<.001)$ (51). Although the impact of RT on local control is convincing, it remains uncertain whether this translates into superior OS.

Many studies have found favorable OS for patients who undergo a resection of BRPC after neoadjuvant chemo(radio)therapy (52). However, some studies overestimated OS with neoadjuvant treatment, because OS was only reported for patients who underwent a curative-intent resection after neoadjuvant treatment, whereas patients who had progressive disease before resection were excluded. A recent meta-analysis resolved this selection bias by including only studies that adhered to the intention-to-treat principle: all patients who started neoadjuvant treatment were included in the analyses, regardless of whether they underwent a resection (50). Only $3 \%$ of these patients received neoadjuvant FOLFIRINOX. The authors found a superior median OS for any neoadjuvant approach (18.8 months) compared with upfront surgery (14.8 months) in (B)RPC patients. In 2018, the first two RCTs for neoadjuvant treatment of (B)RPC completed accrual $(51,53)$. A 
Korean trial was closed prematurely when interim analysis found a superior median OS of 21 months for neoadjuvant CRT vs 12 months with upfront surgery and adjuvant CRT $(P=.028)$ (53). The previously mentioned interim analysis of the PREOPANC-1 trial found a median OS of 17.1 months with neoadjuvant gemcitabine-based CRT vs 13.7 months with upfront surgery and adjuvant gemcitabine $(P=.074)(51)$. However, neither RCT investigated neoadjuvant FOLFIRINOX.

Because FOLFIRINOX is a more effective regimen than gemcitabine alone in the metastatic setting, it is expected that it further improves OS for patients with (B)RPC in the neoadjuvant setting. Four RCTs evaluating neoadjuvant FOLFIRINOX are currently accruing patients: the phase II ALLIANCE A021501 trial (NCT02839343) initially compared neoadjuvant mFOLFIRINOX with or without hypofractioned radiation therapy for BRPC, but recently closed the radiation therapy arm because it met the predetermined futility boundary for R0 resection (54); the phase III NorPACT-1 trial (NCT02919787) for resectable pancreatic cancer comparing neoadjuvant FOLFIRINOX with upfront surgery, both followed by adjuvant gemcitabine and capecitabine (55); the phase II PANACHE01-PRODIGE48 trial (NCT02959879) for resectable pancreatic cancer comparing neoadjuvant FOLFIRINOX with neoadjuvant FOLFOX chemotherapy and upfront surgery, all followed by adjuvant chemotherapy (56); and the phase III PREOPANC-2 trial (NTR7292) comparing neoadjuvant FOLFIRINOX with neoadjuvant gemcitabine-based CRT for (B)RPC (57). Final results of these trials are not anticipated within the next 5 years. Meanwhile, experienced centers continue to publish impressive results in retrospective studies of neoadjuvant FOLFIRINOX for BRPC and LAPC. Most recently, Michelakos et al. published the updated series of Massachusetts General Hospital. For the combined group of BRPC and LAPC patients who underwent a resection after neoadjuvant FOLFIRINOX, they found a median OS of 37.7 months from diagnosis (58).

Median OS estimates after neoadjuvant treatment for BRPC may appear inferior to outcomes with adjuvant chemotherapy. For example, the ESPAC-4 trial reported a median OS of 28.0 months in the adjuvant gemcitabine-capecitabine arm (59). Moreover, the recent PRODIGE 24/CCTG PA.6 trial reported a median OS of 54.4 months in the adjuvant FOLFIRINOX arm (60). However, the patient populations of a neoadjuvant trial and an adjuvant trial are highly different and cannot be compared directly. To be eligible for an adjuvant trial, a patient has to overcome several hurdles. A small percentage of patients will never make it to the operating room, often because of a combination of drainage-related complications (eg, cholangitis or pancreatitis) and frailty. Moreover, about $20 \%$ of radiographically BRPC patients will never undergo resection because of occult metastatic disease at staging laparoscopy or unexpected LAPC during surgical exploration $(61,62)$. Finally, most adjuvant trials require a complete macroscopic resection, a CA 19-9 level below 180 U/ $\mathrm{mL}$, and full recovery from surgery within 12 weeks after resection. In large nationwide studies, only about $55 \%$ of patients received adjuvant chemotherapy (4-6). Neoadjuvant trials include all those patients who drop out during treatment; only about one-third of these patients would be eligible for adjuvant trials after undergoing a resection and remaining fit for adjuvant chemotherapy. Excluding the worst two-thirds of patients will obviously have a major impact on the median OS.

After neoadjuvant FOLFIRINOX, resection rates ranged from $50 \%$ to $100 \%$ across studies. This substantial heterogeneity may be explained by the lack of consensus regarding resectability criteria and criteria to proceed with surgery after neoadjuvant treatment. Reaching consensus on resectability criteria is needed to improve comparison in future studies. In the pooled analysis of the present study, we found a resection rate of $68 \%$. A similar resection rate of $66 \%$ was found in an intention-totreat meta-analysis of (B)RPC patients treated with any neoadjuvant CRT regimen (50). The pooled R0 resection rate of $84 \%$ in the present study was higher compared with the intention-totreat $\mathrm{R} 0$ resection rate of $67 \%$ with upfront surgery (50).

Some limitations of the present study should be considered. Although the present study represents the best available estimate of survival after neoadjuvant FOLFIRINOX for BRPC patients, it might be an overestimate because of the retrospective nature of most included studies. Similarly, secondary study endpoints such as AEs and PFS were prone to selection and information bias. Heterogeneity across studies also might have biased the results; studies used different resectability criteria, FOLFIRINOX regimens, and additional treatment (eg, CRT).

In conclusion, this patient-level meta-analysis of BRPC patients treated with neoadjuvant FOLFIRINOX showed a favorable median OS, resection rate, and $\mathrm{RO}$ resection rate. These results need to be assessed in a randomized trial.

\section{Funding}

This work was supported by the Dutch Cancer Society (grant 2017-109555) and The Netherlands Organisation for Health Research and Development (grant 2017-8430041 08).

\section{Notes}

Affiliations of authors: Department of Surgery, Erasmus MC University Medical Center, Rotterdam, the Netherlands (QPJ, SB, MS, BRB, CHJVE, BGK); Hepato-Pancreato-Biliary Surgery and Liver Transplantation, Pôle des Pathologies Digestives, Hépatiques et de la Transplantation, Hôpital de HautepierreHôpitaux Universitaires de Strasbourg, Strasbourg, France (PA, $\mathrm{PB})$; Department of Oncology, University of Pittsburgh Medical Center, Pittsburgh, PA (NB); Department of Oncology, Mayo Clinic, Phoenix, AZ (TB-S); Radiology Department, Royal Marsden NHS Foundation Trust, Sutton, UK (MAB); Department of Surgery, Cancer Center Amsterdam, Amsterdam UMC, University of Amsterdam, Amsterdam, the Netherlands (MGB, EvV); Department of Surgery, West Virginia University, Morgantown, WV (BAB); Department of Medicine, The Royal Marsden NHS Foundation Trust, London and Surrey, UK (IC); Department of Oncology, Royal North Shore Hospital, Sydney, NSW, Australia (SC); Sydney Medical School (Northern), The University of Sydney, Northern Cancer Institute, Sydney, NSW, Australia (SC); Division of Surgical Oncology, the Ohio State University Wexner Medical Center and James Comprehensive Cancer Center, Columbus, OH (MD); Winship Cancer Institute, Emory University, Atlanta, GA (BFE-R); Department of Radiation Oncology, H Lee Moffitt Cancer Center \& Research Institute, Tampa, FL (JMF); West of Scotland Pancreatic Unit, Glasgow Royal Infirmary, Glasgow, UK (DG, NBJ, CJM); Department of Medicine, University of Miami Miller School of Medicine, Miami, FL (PJH); Wolfson Wohl Cancer Research Centre, Institute of Cancer Sciences, University of Glasgow, Glasgow, UK (NBJ, CJM); Department of Surgery, Johns Hopkins Hospital, Baltimore, MD (AAJ, GAM, NAP, MJW); Department of Oncology, Asan Medical Center, University of Ulsan College of Medicine, Seoul, Korea (KPK); Department of Surgery, Asan Medical Center, University of Ulsan College of Medicine, Seoul, Korea (SCK); Division of Hematology and Oncology, University of California, San 
Francisco, CA (SSK, AHK); Department of Medicine, Yale Cancer Center, Yale School of Medicine, New Haven, CT (JL); Department of Surgery, University of Colorado School of Medicine, Aurora, CO (MDM, AP); Department of Radiation Oncology, University of Miami, Miller School of Medicine, Sylvester Comprehensive Cancer Center, Miami, FL (EAM); Second Department of Surgery, Wakayama Medical University, Wakayama, Japan (K-IO, HY); Department of Radiation Oncology, Henry Ford Cancer Institute, Detroit, MI (PJP); Department of Surgery, LKH Hochsteiermark-Leoben, Leoben, Austria (HR); Upper GI Surgical Unit, Department of Gastrointestinal Surgery, Royal North Shore Hospital, Sydney, NSW, Australia (JS); Sydney Medical School (Northern), The University of Sydney, Australia (JS); Department of HematoOncology, LKH Hochsteiermark-Leoben, Leoben, Austria (CT); Department of Radiotherapy, Cancer Center Amsterdam, Amsterdam UMC, University of Amsterdam, Amsterdam, the Netherlands (GvT); Department of Oncology, Washington University, St. Louis, MO (AW-G); Department of Medical Oncology, Cancer Center Amsterdam, Amsterdam UMC, University of Amsterdam, Amsterdam, the Netherlands (JWW); Department of Medical Oncology, Erasmus MC University Medical Center, Rotterdam, the Netherlands (MYVH); Department of Surgery, MD Anderson Cancer Center, Houston, TX (MHGK).

The funders had no role in the design of the study; the collection, analysis, and interpretation of the data; the writing of the manuscript; and the decision to submit the manuscript for publication.

MD received educational support from Intuitive; EAM received travel funding from ViewRay; SC received travel funding from AstraZeneca, Roche, BMS, and Ipsen and is a member of the advisory boards of AstraZeneca, Roche, BMS, Ipsen, and Specialised Therapeutics Australia; PJH received research funding from AstraZeneca and Kite and is a member of the advisory boards of Ipsen and Angiodynamics; BFE-R received research funding from Boston Biomedical, Bayer, Pfizer, Novartis, Merck, BMS, and Roche and serves as a consultant for Astellas, Taiho, Ipsen, Novartis, Lexicon, and Roche; AW-G serves as consultant for Tyme; MM received research funding from Merck; SCK received research funding from the Korean Health Technology R\&D Project, Ministry of Health \& Welfare, Republic of Korea (HI14C2640). The other authors have no competing interests to declare.

\section{References}

1. Rahib L, Smith BD, Aizenberg R, et al. Projecting cancer incidence and deaths to 2030: The unexpected burden of thyroid, liver, and pancreas cancers in the United States. Cancer Res. 2014;74(11):2913-2921.

2. Cancer Research UK: Pancreatic cancer incidence statistics. https://www cancerresearchuk.org/health-professional/cancer-statistics/statistics-bycancer-type/pancreatic-cancer/incidence\#ref-3. Accessed August 5, 2018.

3. Paniccia A, Merkow J, Edil BH, et al. Immunotherapy for pancreatic ductal ad enocarcinoma: an overview of clinical trials. Chin J Cancer Res. 2015;27(4) 376-391.

4. Merkow RP, Bilimoria KY, Tomlinson JS, et al. Postoperative complications reduce adjuvant chemotherapy use in resectable pancreatic cancer. Ann Surg. 2014:260(2):372-377.

5. Mayo SC, Gilson MM, Herman JM, et al. Management of patients with pancreatic adenocarcinoma: national trends in patient selection, operative management, and use of adjuvant therapy. J Am Coll Surg. 2012;214(1):33-45.

6. IKNL. Report on pancreatic and periampullary carcinoma in the Netherlands https://www.iknl.nl/docs/default-source/KIB-rapportages/portfolio_kib_ pancreas-en-periamplullair-carcinoom.pdf 2014. Accessed December 3, 2018

7. Sohal DP, Walsh RM, Ramanathan RK, et al. Pancreatic adenocarcinoma: treating a systemic disease with systemic therapy. J Natl Cancer Inst. 2014 106(3):dju011.
8. O'Reilly EM, Perelshteyn A, Jarnagin WR, et al. A single-arm, nonrandomized phase II trial of neoadjuvant gemcitabine and oxaliplatin in patients with resectable pancreas adenocarcinoma. Ann Surg. 2014;260(1):142-148.

9. Varadhachary GR, Wolff RA, Crane $\mathrm{CH}$, et al. Preoperative gemcitabine and cisplatin followed by gemcitabine-based chemoradiation for resectable adenocarcinoma of the pancreatic head. J Clin Oncol. 2008;26(21):3487-3495.

10. Evans DB, Varadhachary GR, Crane $\mathrm{CH}$, et al. Preoperative gemcitabine-based chemoradiation for patients with resectable adenocarcinoma of the pancreatic head. J Clin Oncol. 2008;26(21):3496-3502.

11. Conroy T, Desseigne F, Ychou M, et al. FOLFIRINOX vs gemcitabine for metastatic pancreatic cancer. N Engl J Med. 2011;364(19):1817-1825.

12. Pietrasz D, Marthey L, Wagner $\mathrm{M}$, et al. Pathologic major response after FOLFIRINOX is prognostic for patients secondary resected for borderline or locally advanced pancreatic adenocarcinoma: an AGEO-FRENCH, prospective, multicentric cohort. Ann Surg Oncol. 2015;22(S3):1196-1205.

13. Katz MH, Shi Q Ahmad SA, et al. Preoperative modified FOLFIRINOX treatment followed by capecitabine-based chemoradiation for borderline resectable pancreatic cancer: Alliance for Clinical Trials in Oncology trial A021101. JAMA Surg. 2016;151(8):e161137

14. Mellon EA, Hoffe SE, Springett GM, et al. Long-term outcomes of induction chemotherapy and neoadjuvant stereotactic body radiotherapy for borderline resectable and locally advanced pancreatic adenocarcinoma. Acta Oncol. 2015;54(7):979-985.

15. Ferrone CR, Marchegiani G, Hong TS, et al. Radiological and surgical implications of neoadjuvant treatment with FOLFIRINOX for locally advanced and borderline resectable pancreatic cancer. Ann Surg. 2015;261(1):12-17.

16. Liberati A, Altman DG, Tetzlaff J, et al. The PRISMA statement for reporting systematic reviews and meta-analyses of studies that evaluate healthcare interventions: explanation and elaboration. BMJ. 2009;339:b2700.

17. Peddi PF, Cho M, Wang J, et al. Nab-paclitaxel monotherapy in refractory pancreatic adenocarcinoma. J Gastrointest Oncol. 2013;4(4):370-373.

18. Shrestha B, Sun YF, Faisal F, et al. Long-term survival benefit of upfront chemotherapy in patients with newly diagnosed borderline resectable pancreatic cancer. Cancer Med. 2017;6(7):1552-1562.

19. Grose D, McIntosh D, Jamieson N, et al. The role of induction chemotherapy + chemoradiotherapy in localised pancreatic cancer: initial experience in Scotland. J Gastrointest Oncol. 2017;8(4):683-695.

20. Kim SS, Nakakura EK, Wang ZJ, et al. Preoperative FOLFIRINOX for borderline resectable pancreatic cancer: is radiation necessary in the modern era of chemotherapy? J Surg Oncol. 2016;114(5):587-596.

21. Cassinotto C, Cortade J, Belleannee G, et al. An evaluation of the accuracy of CT when determining resectability of pancreatic head adenocarcinoma after neoadjuvant treatment. Eur J Radiol. 2013;82(4):589-593.

22. Egger M, Davey Smith G, Schneider M, et al. Bias in meta-analysis detected by a simple, graphical test. BMJ. 1997;315(7109):629-634.

23. Higgins JP, Thompson SG, Deeks JJ, et al. Measuring inconsistency in metaanalyses. BMJ. 2003;327(7414):557-560.

24. Cochrane handbook for systematic reviews of interventions: identifying and measuring heterogeneity. https://handbook-5-1.cochrane.org/chapter_9/9 5_2_identifying_and_measuring_heterogeneity.htm. Accessed October 11, 2018

25. Paniccia A, Edil BH, Schulick RD, et al. Neoadjuvant FOLFIRINOX application in borderline resectable pancreatic adenocarcinoma: a retrospective cohort study. Medicine (Baltimore). 2014;93(27):e198.

26. Christians KK, Tsai S, Mahmoud A, et al. Neoadjuvant FOLFIRINOX for borderline resectable pancreas cancer: a new treatment paradigm? Oncologist 2014;19(3):266-274

27. Okada K, Kawai M, Hirono S, et al. Impact of treatment duration of neoadjuvant FIRINOX in patients with borderline resectable pancreatic cancer: a pilot trial. Cancer Chemother Pharmacol. 2016;78(4):719-726.

28. Shaib WL, Hawk N, Cassidy RJ, et al. A phase 1 study of stereotactic body radiation therapy dose escalation for borderline resectable pancreatic cancer af ter modified FOLFIRINOX (NCT01446458). Int J Radiat Oncol Biol Phys. 2016 96(2):296-303.

29. Yoo C, Kang J, Kim KP, et al. Efficacy and safety of neoadjuvant FOLFIRINOX for borderline resectable pancreatic adenocarcinoma: improved efficacy compared with gemcitabine-based regimen. Oncotarget. 2017;8(28):46337-46347.

30. Itchins M, Arena J, Nahm CB, et al. Retrospective cohort analysis of neoadjuvant treatment and survival in resectable and borderline resectable pancreatic ductal adenocarcinoma in a high volume referral centre. Eur J Surg Oncol. 2017;43(9):1711-1717.

31. Boone BA, Steve J, Krasinskas AM, et al. Outcomes with FOLFIRINOX for borderline resectable and locally unresectable pancreatic cancer. J Surg Oncol. 2013;108(4):236-241.

32. Addeo P, Rosso E, Fuchshuber P, et al. Resection of borderline resectable and locally advanced pancreatic adenocarcinomas after neoadjuvant chemotherapy. Oncology. 2015;89(1):37-46.

33. Khushman M, Dempsey N, Cudris Maldonado J, et al. Full dose neoadjuvant FOLFIRINOX is associated with prolonged survival in patients with locally advanced pancreatic adenocarcinoma. Pancreatology. 2015;15(6):667-673.

34. Blazer M, Wu C, Goldberg RM, et al. Neoadjuvant modified (m) FOLFIRINOX for locally advanced unresectable (LAPC) and borderline resectable (BRPC) adenocarcinoma of the pancreas. Ann Surg Oncol. 2015;22(4):1153-1159. 
35. Badiyan SN, Olsen JR, Lee AY, et al. Induction chemotherapy followed by concurrent full-dose gemcitabine and intensity-modulated radiation therapy for borderline resectable and locally advanced pancreatic adenocarcinoma. Am J Clin Oncol Cancer Clin Trials. 2016;39(1):1-7.

36. Vogel JA, Rombouts SJ, de Rooij T, et al. Induction chemotherapy followed by resection or irreversible electroporation in locally advanced pancreatic cancer (IMPALA): a prospective cohort study. Ann Surg Oncol. 2017;24(9): 2734-2743.

37. Tinchon C, Hubmann E, Pichler A, et al. Safety and efficacy of neoadjuvant FOLFIRINOX treatment in a series of patients with borderline resectable pancreatic ductal adenocarcinoma. Acta Oncol. 2013;52(6):1231-1234.

38. Peddi PF, Lubner S, McWilliams R, et al. Multi-institutional experience with FOLFIRINOX in pancreatic adenocarcinoma. JOP. 2012;13(5):497-501.

39. Mahaseth H, Brutcher E, Kauh J, et al. Modified FOLFIRINOX regimen with improved safety and maintained efficacy in pancreatic adenocarcinoma. Pancreas. 2013;42(8):1311-1315

40. Moorcraft SY, Khan K, Peckitt C, et al. FOLFIRINOX for locally advanced or metastatic pancreatic ductal adenocarcinoma: The Royal Marsden experience. Clin Colorectal Cancer. 2014;13(4):232-238.

41. Stein SM, James ES, Deng Y, et al. Final analysis of a phase II study of modified FOLFIRINOX in locally advanced and metastatic pancreatic cancer. $\mathrm{Br} \mathrm{J}$ Cancer. 2016;114(7):737-743.

42. NCCN. Pancreatic adenocarcinoma, version 2.2017, clinical practice guidelines in oncology. J Natl Compr Cancer Netw. 2017;15(8):1028-1061.

43. Callery MP, Chang KJ, Fishman EK, et al. Pretreatment assessment of resectable and borderline resectable pancreatic cancer: expert consensus statement. Ann Surg Oncol. 2009;16(7):1727-1733.

44. Katz MH, Marsh R, Herman JM, et al. Borderline resectable pancreatic cancer: need for standardization and methods for optimal clinical trial design. Ann Surg Oncol. 2013;20(8):2787-2795.

45. Varadhachary GR, Tamm EP, Abbruzzese JL, et al. Borderline resectable pancreatic cancer: definitions, management, and role of preoperative therapy. Ann Surg Oncol. 2006;13(8):1035-1046.

46. Susan Williamson DG, Barbour A, Samra J, Haghighi K, Nikfarjam M, Kench J, on behalf of the Australasian Gastro-Intestinal Trials Group (AGITG) Definition of surgical standards for pancreatic cancer: a consensus statement by the Australasian Gastro-Intestinal Trials Group. https://gicancer.org.au/ news/surgical-standards-for-pancreatic-cancer-consensus-statement/.

47. DPCG. Table 1: CT staging for adenocarcinoma of the pancreatic head and uncinate process (DPCG, 2012). http://dpcg.nl/images/Criteria_resectabiliteit. pdf. Accessed April 2, 2018.

48. Conroy T, Paillot B, Francois E, et al. Irinotecan plus oxaliplatin and leucovorin-modulated fluorouracil in advanced pancreatic cancer-a Groupe Tumeurs Digestives of the Federation Nationale des Centres de Lutte Contre le Cancer study. J Clin Oncol. 2005;23(6):1228-1236.

49. Suker M, Beumer BR, Sadot E, et al. FOLFIRINOX for locally advanced pancreatic cancer: a systematic review and patient-level meta-analysis. Lancet Oncol. 2016;17(6):801-810.
50. Versteijne E, Vogel JA, Besselink MG, et al. Meta-analysis comparing upfront surgery with neoadjuvant treatment in patients with resectable or borderline resectable pancreatic cancer. BrJ Surg. 2018;105(8):946-958.

51. van Tienhoven G, Versteijne E, Suker M, et al. Preoperative chemoradiother apy vs immediate surgery for resectable and borderline resectable pancreatic cancer (PREOPANC-1): a randomized, controlled, multicenter phase III trial. J Clinc Oncol. 2018;36(18):LBA4002-LBA4002.

52. Gillen S, Schuster T, Meyer Zum BC, et al. Preoperative/neoadjuvant therapy in pancreatic cancer: a systematic review and meta-analysis of response and resection percentages. PLoS Med. 2010;7(4):e1000267.

53. Jang JY, Han Y, Lee $\mathrm{H}$, et al. Oncological benefits of neoadjuvant chemoradiation with gemcitabine vs upfront surgery in patients with borderline resectable pancreatic cancer: a prospective, randomized, open-label, multicenter phase 2/3 trial. Ann Surg. 2018;268(2):215-222.

54. Katz MHG, Ou FS, Herman JM, et al. Alliance for clinical trials in oncology (ALLIANCE) trial A021501: preoperative extended chemotherapy vs chemotherapy plus hypofractionated radiation therapy for borderline resectable adenocarcinoma of the head of the pancreas. BMC Cancer. 2017;17(1):505.

55. Labori KJ, Lassen K, Hoem D, et al. Neoadjuvant chemotherapy vs surgery first for resectable pancreatic cancer (Norwegian Pancreatic Cancer Trial-1 (NorPACT-1))-study protocol for a national multicentre randomized controlled trial. BMC Surg. 2017;17(1):94.

56. Schwarz L, Vernerey D, Bachet JB, et al. Resectable pancreatic adenocarcinoma neo-adjuvant FOLF(IRIN)OX-based chemotherapy - a multicenter, non-comparative, randomized, phase II trial (PANACHE01-PRODIGE48 study). BMC Cancer. 2018;18(1):762.

57. Janssen QP, Besselink MG, Wilmink JW, van Tienhoven G, Homs M, Groot Koerkamp B, on behalf of the Dutch Pancreatic Cancer Group. The (cost)effectiveness of neoadjuvant FOLFIRINOX us neoadjuvant gemcitabine-based chemoradiotherapy and adjuvant gemcitabine for (borderline) resectable pancreatic cancer: the PREOPANC-2 study. In: 13th IHPBA World Congress. September 7, 2018. Geneva, Switzerland; 2018.

58. Michelakos T, Pergolini I, Castillo CF, et al. Predictors of resectability and survival in patients with borderline and locally advanced pancreatic cancer who underwent neoadjuvant treatment with FOLFIRINOX. Ann Surg. 2019;269(4): 733-740.

59. Neoptolemos JP, Palmer DH, Ghaneh P, et al. Comparison of adjuvant gemcitabine and capecitabine with gemcitabine monotherapy in patients with resected pancreatic cancer (ESPAC-4): a multicentre, open-label, randomised, phase 3 trial. Lancet. 2017;389(10073):1011-1024.

60. Conroy T, Hammel P, Hebbar M, et al. FOLFIRINOX or gemcitabine as adjuvant therapy for pancreatic cancer. N Engl J Med. 2018;379(25):2395-2406.

61. Somers I, Bipat S. Contrast-enhanced CT in determining resectability in patients with pancreatic carcinoma: a meta-analysis of the positive predictive values of CT. Eur Radiol. 2017;27(8):3408-3435.

62. Peng JS, Mino J, Monteiro R, et al. Diagnostic laparoscopy prior to neoadjuvant therapy in pancreatic cancer is high yield: an analysis of outcomes and costs. J Gastrointest Surg. 2017;21(9):1420-1427. 